Check for updates

Cite this: J. Mater. Chem. B, 2019, 7, 5328

Received 14th May 2019 ,

Accepted 31st July 2019

DOI: 10.1039/c9tb00965e

rsc.li/materials-b

\section{Flexible, microstructured surfaces using chitin-derived biopolymers $\dagger$}

\author{
Sayantan Pradhan, ${ }^{a}$ Kathryn M. Moore, ${ }^{b}$ Kristy M. Ainslie ${ }^{(D)}{ }^{b}$ and \\ Vamsi K. Yadavalli (D)*a
}

\begin{abstract}
Chitin, one of the most abundant natural amino polysaccharides, is obtained primarily from the exoskeletons of crustaceans, crabs and shrimp. Chitin and its derivative chitosan have gained much attention in the field of biomedical research due to attractive properties such as biocompatibility, non-toxicity, biodegradability, low immunogenicity, and ease of availability. While work has been done on the use of chitin and chitosan as functional biomaterials by imparting specific properties, the potential of chitin as a biomaterial is somewhat limited owing to its intractable processing. In this work, we propose a facile reaction to modify the chitin chain with photoactive moieties for the realization of photocrosslinkable chitin. This chitin derivative is easily usable with a benign solvent formic acid to be able to form mechanically robust, optically transparent sheets. These films exhibit comparable tensile properties to that of native chitin and chitosan and better surface wettability. Most importantly, this material can be used to form precise, high resolution microarchitectures on both rigid and flexible substrates using a facile bench top photolithography technique. These flexible micropatterned 2D sheets of chitin were demonstrated as a dynamic cell culture substrate for the adhesion and proliferation of fibroblasts, wherein the chitin micropatterns act as a template for spatial guidance of cells. This chitin-based biopolymer can find diverse uses in tissue engineering as well as to form components for degradable bioelectronics.
\end{abstract}

\section{Introduction}

Chitin is the most abundant aminopolysaccharide in nature, found mainly as ordered fibrils in the exoskeleton of arthropods. ${ }^{1}$ Together with its most common derivative, the partially deacetylated form - chitosan, it is an important material for biomedical applications due to attractive properties such as biocompatibility, non-toxicity, biodegradability, low immunogenicity, and ease of availability. ${ }^{2,3}$ The presence of labile functional groups on the main chains of chitin and chitosan provides an opportunity to improve the material properties and function by ligating different chemical moieties. ${ }^{4-6}$ Excellent biological properties and novel intrinsic antimicrobial properties have favored the use of chitin and its derivatives in tissue engineering, wound healing, and drug delivery applications. ${ }^{7,8}$ To develop functional architectures

\footnotetext{
${ }^{a}$ Department of Chemical and Life Science Engineering, Virginia Commonwealth University, Richmond, VA 23284, USA. E-mail:vyadavalli@vcu.edu

${ }^{b}$ Pharmacoengineering \& Molecular Pharmaceutics, UNC Eshelman School of Pharmacy, Chapel Hill, NC 27599, USA

$\dagger$ Electronic supplementary information (ESI) available: Synthesis details, characterization including measurement of degree of deacetylation, FTIR spectra and analysis, prior literature on tensile testing of both chitin and chitosan films, and additional images of the films and microstructures formed using this biomaterial and technique. See DOI: 10.1039/c9tb00965e
}

from chitin and chitosan, the polymers are frequently dissolved acidic solutions. ${ }^{9}$ Using this approach, there have been reports on the use of chitinous biomaterials for porous biodegradable scaffolds, with the polymers alone, ${ }^{10,11}$ or in combination with other materials such as gelatin, collagen, poly-L-lactic acid, alginate, and silk. ${ }^{12-18}$ With the development of new processing chemistries, chitin and its derivatives have been used as cell culture platforms in the form of hydrogels, membranes, films and 3D structures. ${ }^{19-22}$ A majority of prior work on this family of biomaterials has been focused on chitosan and not on chitin, owing to the limited solubility of the latter. ${ }^{1}$ With an annual worldwide chitin production of approximately $10^{11}$ tons, the production cost of chitosan is higher as the deacetylated form of chitin requires additional enzymatic or chemical hydrolysis. ${ }^{3}$

To date, only a few strategies to form chitin into precise $2 \mathrm{D}$ and 3D micropatterned structures with high resolution have been reported. Much research has been focused on forms such as chitin nanocrystals (CHNC) and chitin nanofibers (CHNF). ${ }^{23}$ Micro and nanopatterned biomaterial surfaces are increasingly important both as cell culture substrates as well as components of biosensors and organic electronics. ${ }^{24,25}$ To this end, fabrication techniques have been reported for the realization of chitin/chitosan microstructures as templates for guided cell growth. A nanosphere lithography (NSL) technique using silver nanoparticle templates was 
used to form flexible nanostructured chitin-mats. ${ }^{26}$ This process forms nanostructures on chitin wing substrates guided by the hexagonal close packing organization of nanoparticles. Nanostructured chitin membranes and papers have been demonstrated. ${ }^{27,28}$ Micromolding was applied to photocrosslinkable chitosan to develop spatially controlled 3D co-culture systems. ${ }^{29}$ This system was combined with photolithography to create microstructures on tissue culture polystyrene (TCP) and glass. ${ }^{30}$ Soft lithography was used with chitosan to create a $2 \mathrm{D}$ microstructured surface, which in turn was used to construct 3D cell culture surfaces. ${ }^{31}$ Indirect patterning using chitosan has also been shown, wherein a random copolymer of oligo(ethylene glycol)methacrylate (OEGMA) and methacrylic acid (MA) was patterned on a chitosan substrate. ${ }^{32}$ Other patterning for cell alignment has been shown via micromolding, ${ }^{33}$ inkjet printing using a chitinous nanocrystal ink, ${ }^{34}$ and macro scale molding and micro-contact patterning with a chitin nanofiber ink. ${ }^{35}$ Some of these fabrication processes require high resolution molds which have issues of transfer and fidelity in providing scalable and tunable spatial conformations. As yet, creating structures of high resolution using scalable techniques such as photolithography has not been demonstrated with chitin. Though there has been some work on micro-patterning, primarily on rigid substrates, chitin micropattering on flexible surfaces can open new avenues for its use. The ability to form precise high resolution microstructures of chitin, while using scalable techniques in different formats (2D, 3D and with flexible substrates) can provide a new strategy to form bioelectronics devices that are biofriendly and degradable. ${ }^{36}$ Optically transparent, flexible, micropatterned chitin sheets can be used for green electronics, tissue engineering, or to achieve conformal contact for wound healing applications. ${ }^{37}$

In this work, a facile reaction for the modification of chitin to produce a photocrosslinkable chitin (PC) is demonstrated. The "photochitin" is distinct from earlier reports of photocrosslinkable chitosan $^{38}$ in being able to be directly utilize chitin as a starting material, as well as other UV-crosslinked hydrogels formed from chitin-methacrylate. ${ }^{39,40}$ To the best of our knowledge, this is the first report of a chitin material that can be used to form micropatterned surfaces. The photocrosslinkable chitin can be easily formed into free-standing sheets (plain and patterned) with excellent tensile properties using a benign solvent, formic acid. The utilization of such techniques is quite attractive since they do not require clean room facilities or harsh chemicals. Using photolithographic tools, the ability of this material to fabricate high resolution structures on both rigid and flexible substrates, suitable for cell adhesion and proliferation is shown. Micro-patterned sheets are shown to provide contact guidance for cells, an essential first step towards forming functional tissue. These biodegradable substrates can potentially allow the formation of patterned cell sheets without any (rigid) supports. Intrinsically photoreactive chitin derivatives also represent a versatile material that may be employed in conjunction with modern prototyping tools towards 3D architectures. As a biomaterial that can be widely recovered from a variety of sources including marine organisms, novel chitin derivatives can form a versatile and sustainable precursor for diverse applications.

\section{Experimental section}

\subsection{Synthesis of low molecular weight chitin}

In order to synthesize photocrosslinkable chitin, it was necessary to convert the raw chitin flakes into low molecular weight chitin (LMW chitin). LMW chitin was prepared using a well-established method. ${ }^{41}$ In brief, $60 \mathrm{~mL}$ of $37 \% \mathrm{HCl}$ was added to $2 \mathrm{~g}$ chitin (Alfa Aesar, Haverhill, MA) in a round bottom flask. The mixture was heated at $40{ }^{\circ} \mathrm{C}$ using a water batch and continuously stirred for $\sim 20$ minutes. The contents of the flask were cooled using an ice-bath, and then carefully neutralized to a $\mathrm{pH} 7$ using $\mathrm{NaOH}$ solution. The mixture was then centrifuged at $4000 \mathrm{rpm}$ for 20 minutes and the supernatant was collected. This solution was then dialyzed against tap water using $12 \mathrm{kDa}$ dialysis membrane for 2 days, with periodic replacement of the tap water. The solution within the dialysis tubing was collected and lyophilized for 2 days to obtain low molecular weight chitin powder.

\subsection{Synthesis and characterization of photocrosslinkable chitin}

Photocrosslinkable chitin (which refer to as photochitin (PC)) was prepared by adding photoactive moieties to the chitin backbone. All glassware was cleaned using deionized water and ethanol, and oven dried at $150{ }^{\circ} \mathrm{C}$ overnight. All the chemicals were dried to ensure they were free from any moisture. Low molecular weight chitin was dissolved in $5 \% \mathrm{LiCl} /$ dimethylacetamide (DMAc) in a 3 neck round bottom flask and 2-isocyanatoethyl methacrylate (IEM) was added to the reaction mixture in excess. The reaction was carried out at $65{ }^{\circ} \mathrm{C}$ for 24 hours while maintaining inert conditions using a continuous flow of nitrogen. The product obtained was precipitated overnight in cold ethanol, followed by 3 cycles of washing using 1:1 ratio of cold ethanol and acetone. The product was lyophilized to obtain PC powder. The yield of the product was $\sim 54 \%$. The degree of deacetylation (DD), which determines the content of free amino groups in the polysaccharides was then used to determine the chemical nature of the photochitin (PC) synthesized. Typically, chitin with a high degree of deacetylation $(>70 \%)$ is classified as chitosan. Various methods have been proposed for the determination of the DD. Some tend to be complex and expensive (e.g. NMR) or destructive to the sample (ninhydrin test). ${ }^{42}$ Therefore, we used two well developed analytical procedures for the DD determination - viz. infrared spectroscopy (IR) and first derivative UV-spectrophotometry for confirmation of the results. ${ }^{43,44}$ Based on our experiments, the DD of the photochitin is estimated to be $\sim 40 \%$, with excellent agreement using both methods. The results were further validated using manufacturer supplied data for pure chitin (20-30\%) and chitosan (75-85\%). The detailed experiments and results are included in the ESI. $\dagger$

\subsection{Fabrication of flexible films}

Films were fabricated by casting a solution of photochitin in formic acid (Acros Organics 98\%) with composition $-40 \mu \mathrm{L}$ of FA, $0.1 \mu \mathrm{L}$ of PEG-DA (Sigma-Aldrich, St. Louis, MO) and $1 \mathrm{mg}$ of photo initiator (2-hydroxy-4'-(2-hydroxyethoxy)-2-methylpropiophenone, Sigma-Aldrich) per $3 \mathrm{mg}$ of PC. The solution was dried under ambient conditions and crosslinked under $365 \mathrm{~nm}$ UV lamp (Lumen Dynamics OmniCure 1000 system) for 2 seconds 
at $20 \mathrm{mM} \mathrm{cm} \mathrm{cm}^{-2}$. Films of pure chitin were fabricated using a process developed elsewhere. ${ }^{45}$ In brief, a suspension of $1 \mathrm{gm}$ chitin powder in $\sim 15 \mathrm{~mL}$ of formic acid was prepared and then frozen at $-20{ }^{\circ} \mathrm{C}$ for 20 hours. The frozen suspension was thawed at room temperature, stirred and frozen again. The process was carried out for several times till a clear gel was obtained. The obtained clear chitin gel was added to small amount of trichloroacetic acid, mixed thoroughly was cast on a clean glass slide. The solvents were evaporated under ambient conditions and a chitin film was obtained. Films of chitosan was obtained by casting a $7.5 \%(\mathrm{w} / \mathrm{v})$ solution of chitosan in formic acid on a clean glass slide and drying it under atmospheric conditions to obtain a film.

\subsection{Characterization of films}

IR was conducted on films of chitin and photochitin to verify the presence of the methacrylate moiety as well as determine the degree of deacetylation as discussed above. Films were cast from a solution in formic acid onto a germanium crystal for analysis. Fourier-transform infrared (FTIR) spectra were obtained at a resolution of $1 \mathrm{~cm}^{-1}$ and 32 scans, via a Nicolet iS10 FTIR spectrometer, equipped with a Smart iTR attachment for attenuated total reflectance (ATR) studies. Contact angles of chitin, photochitin, and chitosan films were measured using a Ramé-Hart goniometer (Succasunna, NJ) by placing a $5 \mu \mathrm{L}$ drop of DI water on the films and taking images with the attached camera. Tensile tests were performed on PC, chitin and chitosan films prepared using methods described above using MTS 300 series tensile testing machine (MTS Systems Corporation, Eden Prairie, MN) equipped with a $50 \mathrm{~N}$ load cell. The tensile measurements were taken under ambient conditions on complete dry films. A strain rate of $0.1 \mathrm{~mm} \mathrm{~s}^{-1}$ was applied on the films and data was collected at a rate of $10 \mathrm{~Hz}$. The tensile strengths of chitin, photochitin, and chitosan films were compared.

\subsection{Fabrication of micropatterns}

Flexible chitin substrate was created using a solution of $2.5 \%$ $(\mathrm{w} / \mathrm{v})$ photochitin and $0.75 \%(\mathrm{w} / \mathrm{v})$ PEG-DA in formic acid (Acros Organics $98 \%$ ). $0.83 \%(\mathrm{w} / \mathrm{v})$ photo initiator (2-hydroxy-4'-(2hydroxyethoxy)-2-methylpropiophenone, Sigma-Aldrich, St. Louis, MO) was added to the solution and the solution was casted on a clean glass slide. The solution was air dried under ambient conditions and then crosslinked under a $365 \mathrm{~nm}$ UV lamp (Lumen Dynamics OmniCure 1000 system) for 2 seconds at $20 \mathrm{~mW} \mathrm{~cm}^{-2}$. The films were soaked in DI water to obtain freestanding chitin films. In order to create micropatterns on flexible chitin substrates, a solution of $2 \%(\mathrm{w} / \mathrm{v})$ photochitin + $0.75 \%(w / v)$ PEG-DA with $0.66 \%$ photo initiator in $1,1,1,3,3,3$ hexafluoro-2-propanol (HFIP, Sigma-Aldrich) was drop cast on the chitin films prepared as above. The samples were air dried for $\sim 5$ minutes and then exposed under the UV lamp for 1 second through a negative tone photomask with desired patterns. The patterns were developed by soaking in a $1 \mathrm{M}$ LiCl/DMSO solution for 1 hour. The micropatterned films and substrates were cleaned using generous amounts of DI water.

\subsection{Cell culture experiments}

All cell culture experiments were performed with NIH 3T3 fibroblasts (ATCC, Manassas, VA). Cells were cultured in Dulbecco's Modified Eagle's Medium (DMEM) supplemented with $10 \%$ fetal bovine serum and $1 \%$ penicillin/streptomycin at $37{ }^{\circ} \mathrm{C}$. Cell seeding efficiency was measured by MTT (3-(4,5-dimethylthiazol-2-yl)-2,5diphenyltetrazolium bromide) assay after cells were seeded onto films for $48 \mathrm{~h}$. A standard curve of cells seeded on tissue culture treated polystyrene of equivalent surface area was used to determine cell number on films. Seeding efficiency determined by normalizing cell number on scaffolds to 10000 cells seeded. Potential cytotoxicity of scaffolds was measured after $24 \mathrm{~h}$. Cells were cultured on the film surface and cytotoxicity was assessed using an LDH Assay Kit (Thermo Scientific, Waltham, MA). Fluorescent images were taken after 25000 cells were seeded onto films for $72 \mathrm{~h}$. Cells were stained with Invitrogen ${ }^{\mathrm{TM}}$ Molecular Probes ${ }^{\mathrm{TM}}$ CellTracker $^{\mathrm{TM}}$ Green CMFDA Dye (Thermo Scientific) and imaged on an EVOS fluorescent microscope (Thermo Scientific).

\subsection{Imaging}

Scanning electron microscopy (SEM) images of the patterns were taken on a Hitachi SU-70 FE-SEM to observe the fidelity of the patterned structures. The patterns were sputtered coated in a $20 \AA$ platinum Denton vacuum cold sputtering system (Moorestown NJ) prior to imaging. Optical images were taken using a Nikon Eclipse microscope.

\section{Results and discussion}

Extensive studies have been made regarding the functionalization of chitin and chitosan to form derivatives with a repertoire of functionalities. ${ }^{3,46}$ The extensive hydrogen bonding between adjacent polymer chains of the predominantly $\beta-(1 \rightarrow 4)$-linked 2-acetamido-2-deoxy-D-glucose have contributed to high rigidity and poor solubility and processability of chitin. Thus, despite its widespread availability and versatile properties, the potential of chitin remains untapped. Indeed, the derivative, chitosan, has gained popularity over chitin due to its solubility in dilute acids, which in turn makes it easier to manipulate. However, chitin tends to have better chemical and thermal stability in comparison to chitosan. ${ }^{47}$ Further, it has been reported that chitin is more compatible with blood, and activates fewer macrophages. ${ }^{48}$ Therefore, finding new ways to harness chitin can provide realization of new applications. Micropatterning methods for chitin and its derivatives have been primarily limited to approaches using multi-step soft lithography, using molds and curing to impart desired patterns. ${ }^{49,50}$ This presents an opportunity to provide an easily patterned material that can undergo facile processing steps to produce microstructures as well as mechanically robust and flexible sheets.

\subsection{Synthesis and characterization of photocrosslinkable chitin}

The presence of multiple pendant functional groups in the main chain of chitin provide opportunities for the introduction of photo-reactive groups, which can impart photo-crosslinkability to native chitin. Using the concepts of modular design, photoreactive 
methacrylate groups were targeted for conjugation due to their prevalence in existing photolithography techniques, ease of use, and possibility for high-resolution, microscale features. ${ }^{51}$ In earlier works, we reported on the use of a similar strategy to form photoreactive silk proteins, which can readily react with commercially available photoinitiators. ${ }^{52}$ Functionalization of chitin and chitosan with photo-crosslinkable groups has been previously reported. A photo-crosslinkable chitosan derivative was formed by introducing azide functional groups. ${ }^{38}$ Acrylategrafted chitin by the reaction of chitin with acrylic acid in the presence of strong sulfuric acid under heterogeneous conditions was used to produce a chitin-based hydrogel for wound dressing applications. ${ }^{40}$ Chitin-methacrylate (CM) was prepared by the reaction of methacrylic acid on chitin in 5\% LiCl/DMAc in the presence of $N, N^{\prime}$-dicyclocarbodiimide and dimethylaminopyridine. ${ }^{39}$ The currently reported strategy therefore involves the modification of chitin to introduce such groups, and further show an easy process to form micropatterns and sheets.

The low solubility of chitin presents a challenge towards easy processing. $5 \% \mathrm{LiCl}$ in DMAc is one of the few solvent systems that can dissolve chitin. ${ }^{53}$ Hydrolysis into lower molecular weight chitin has been found to increase the solubility of chitin in $5 \%$ LiCl/DMAc. ${ }^{54}$ The polymeric chain of chitin can be broken down into smaller oligomeric or monomeric groups either by enzymatic or by acid hydrolysis. In this work, concentrated hydrochloric acid (37\%) was used to hydrolyze chitin without deacetylation or formation of side products. ${ }^{41}$ The acid catalyzed hydrolysis of chitin into low-molecular weight chitin takes place via cleavage of glycosidic linkages. LMW chitin was then modified to photocrosslinkable chitin (PC) using 2-isocyanatoethyl methacrylate (IEM) with LiCl/DMAc as the solvent. The product obtained was precipitated, purified to separate the water-miscible components (DMAc and IEM) and subsequently lyophilized to give PC powder. The PC powder obtained was found to be soluble in formic acid and in polar fluorinated solvents such as 1,1,1,3,3,3-hexafluoro-2propanol (HFIP). As a more benign solvent, the use of formic acid for chitin processing can be an important advantage. ${ }^{55}$ The steps are shown in the ESI, $\dagger$ Fig. S1.

Unpatterned films were prepared by casting an $11 \%(\mathrm{w} / \mathrm{v})$ solution of the PC in formic acid with $2 \%$ photo initiator $(\mathrm{w} / \mathrm{v})$ and crosslinking using $365 \mathrm{~nm}$ UV light. The crosslinked films were found to stable in water as well as solvent systems such as $1 \mathrm{M} \mathrm{LiCl}$ in DMSO and 5\% LiCl in DMAc. In contrast, both pure (native) chitin films and uncrosslinked PC films are soluble in these solvents, indicating the successful incorporation of acrylate moieties in the native chitin chain which are responsible for the crosslinking in the presence of UV light. The conjugation of the acrylate moiety on the chitin chain was verified by analyzing the FT-IR spectra of chitin, uncrosslinked PC film and crosslinked PC film (spectra are presented in Fig. S2, ESI $\dagger$ ). The spectra of uncrosslinked PC film and crosslinked PC film showed peaks at $1720 \mathrm{~cm}^{-1}$, which can be attributed to the methacrylate carbonyl group $(\mathrm{C}=\mathrm{O}$ stretch $)$ and vinyl group $(\mathrm{C}=\mathrm{C}$ stretch which imparts photoreactivity) peaks at $1640 \mathrm{~cm}^{-1}$, both of which are absent in the spectrum of native chitin. There is an increase in the peak intensity of $\mathrm{C}-\mathrm{O}$ stretch at $\sim 1160 \mathrm{~cm}^{-1}$ in PC films when compared to native chitin. Additionally, it can be noted that on comparison of the uncrosslinked PC and crosslinked PC spectra, there is a decrease in intensity at $1640 \mathrm{~cm}^{-1}$ which can be attributed to the consumption of vinyl $\mathrm{C}=\mathrm{C}$ groups upon crosslinking.

\subsection{Formation of optically transparent, mechanically robust chitin films}

The formation of thin and strong films of chitin has been of great interest. However, owing to the highly ordered aggregate structure, extensive hydrogen bonding, and negligible hydrophobic interactions between chitin chains, coupled with its general incompatibility with many solvents, this has been challenging. Indeed, there have been several reports on using different systems such as concentrated acids $\left(\mathrm{H}_{2} \mathrm{SO}_{4}, \mathrm{H}_{3} \mathrm{PO}_{4}\right.$, $\mathrm{HCOOH}$ ), strong and highly polar solvents (e.g., trichloroacetic acid, dichloroacetic acid, hexafluoroisopropyl alcohol (HFIP), methane sulfonic acid, LiCl/dimethylacetamide [DMAc], and LiCl/N-methyl-2-pyrrolidone), and ionic liquids. ${ }^{1,56}$ Different architectures such as films and fibers have been reported, along with nanoparticles and nanofibers. ${ }^{23,57} \mathrm{~A}$ recent report found the use of a more sustainable aqueous $\mathrm{KOH} / \mathrm{urea}$ solution to form films. ${ }^{37}$ In this work, we formed films of chitin in a very facile process using formic acid which is also a benign solvent. These films are optically transparent and mechanically robust and can be formed with varying thicknesses by casting different concentrations of the PC in formic acid. The films can be formed on substrates (e.g. glass or silicon) by spin-coating followed by UV crosslinking (Fig. 1 top). Usefully, the films can be peeled from the substrates to be free-standing. Given the aforesaid structural characteristics of chitin, it was observed that films of $100 \%$ PC were found to be brittle when dry, but malleable when fully hydrated. However, the addition of a minute amount $(0.1 \%)$ of polyethylene glycol diacrylate (PEG-DA) was found to greatly enhance the mechanical properties of the films, even when dry. These films can be easily held and bent without any damage (Fig. 2, all films are $\sim 60 \mu \mathrm{m}$ thick). From the figure. It can be clearly noted that all these materials form optically transparent films, but the pure chitin films are somewhat cloudy. Thin films $\sim 10-20 \mu \mathrm{m}$ thick can be formed that are conformable on dynamic surfaces (e.g. skin) without tearing.

Contact angles of photochitin, chitin and chitosan films were measured using a Ramé-Hart contact angle goniometer by

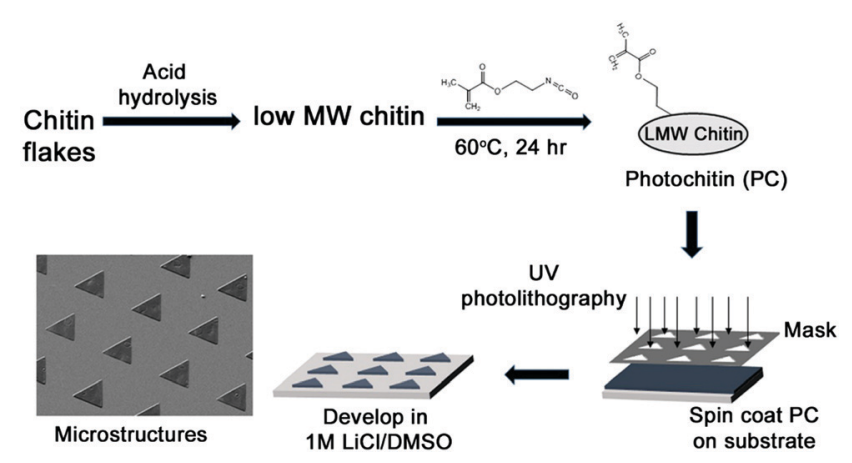

Fig. 1 Schematic showing the synthesis of photochitin (PC) and the fabrication of thin films as well as microstructures of chitin. 


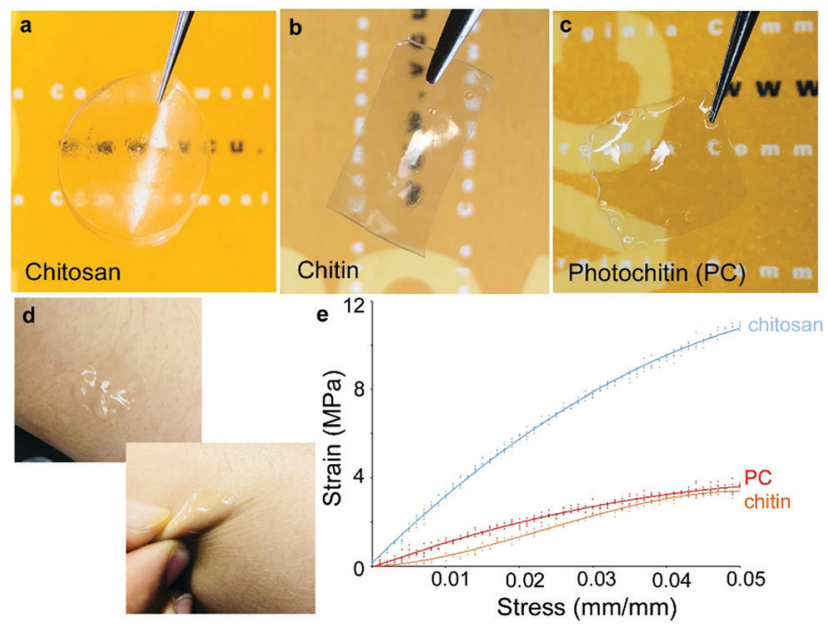

Fig. 2 Mechanically robust and flexible films $\sim 60 \mu \mathrm{m}$ thickness. (a) pure chitosan, (b) pure chitin, (c) photochitin films, all prepared using the same solvent (formic acid). All these films can be easily handled and are optically transparent (photochitin forms the clearest films). (d) At $\sim 10-20 \mu \mathrm{m}$ in thickness can conform to soft interfaces such as skin, where they can be mechanically deformed without breaking. (e) Tensile testing of the films shows that photochitin films are of comparable, if slightly higher strength than films of pure chitin.

placing a $5 \mu \mathrm{L}$ drop of DI water on the films. The advancing contact angle of chitin and chitosan were measured to be around $68.9^{\circ}$ and $90.6^{\circ}$ respectively which is similar to the values reported in literature..$^{58-60}$ Interestingly, the contact angle of photochitin films were measured to be $56.3^{\circ}$ which is hydrophilic in comparison to chitin and chitosan films, and hence showing better surface wettability. On addition of a small amount (0.1\%) PEG to improve mechanical stability, the contact angle is even more hydrophilic at $49.9^{\circ}$. The slight increase in the surface wettability can be attributed to the addition of PEG in the composite (the measurements are shown in the ESI, $\dagger$ Fig. S4). In order to compare their mechanical properties, tensile strength of the PC, chitin and chitosan films, all prepared using a common solvent - formic acid, was tested. The experiment was performed using a $50 \mathrm{~N}$ load cell by applying a strain rate of $0.1 \mathrm{~mm} \mathrm{~s}^{-1}$. The tensile strengths of chitin films were found to be $\sim 66 \mathrm{MPa}$ which is similar to earlier reports. ${ }^{37,61}$ The tensile strength of PC films (with $0.1 \%$ PEG-DA) was measured to be $\sim 90 \mathrm{MPa}$ which indicates that the crosslinking induces a strength higher than the pure chitin films. As a comparison, both films were tested against chitosan and for our experiments, both photochitin and pure chitin films showed a lower tensile strength and elongation in comparison, which is likely owing to their higher structural rigidity. ${ }^{62}$ It is important to note that here, we used a common solvent for all these films (formic acid) to facilitate easy comparison. It has been reported that the properties of chitin and chitosan films are strongly dependent on the preparation conditions and solvents. ${ }^{63,64}$ A table showing the different prior results of tensile testing of chitin and chitosan films is presented in the ESI, $\dagger$ Table S1. While we have not explicitly investigated in this study, earlier we showed that the same photocrosslinking chemistry can be controlled, resulting in films whose mechanical properties and degradation rates can be tuned. Notably, the PC films are optically transparent, mechanically robust, and can be easily fabricated to any desired thickness using a benign solvent, showing their versatility for various applications.

\subsection{Micropatterning of photochitin to form high resolution structures}

Photoreactive chitin provides a versatile biomaterial that can be micropatterned using photolithography, wherein the material acts as a "negative photoresist" crosslinked in the presence of UV light. Removal of unexposed and uncrosslinked material (development step) results in the formation of precise microarchitectures ranging from 10-100 $\mu \mathrm{m}$ (Fig. 2). The scalability of photolithography implies that large areas (several $\mathrm{cm}$ ) with microstructural topology can be easily fabricated, which is difficult using molding or imprinting. SEM imaging was used to characterize surface morphology and fidelity of architectures formed. Expanded views of the topography show the surface at the microscale and the ability to form features of controllable height. Various patterns of different geometry can be easily formed using different photomasks (Fig. 3a and b). Initially, these patterns were formed using functionalized glass and silicon as substrates (rigid substrates). The presence of methacrylate groups on the underlying substrate allows for chemical attachment of the features to the surface, while the uncrosslinked material does not attach and can be removed.

What makes the reported photolithographic technique versatile is that microstructures can be formed on flexible films in order to form micropatterned chitin sheets (Fig. 3c-e). In these experiments, a chitin sheet is initially cast on a substrate followed by crosslinking. A second chitin sheet is cast on the first and on exposure through a photomask, microscale

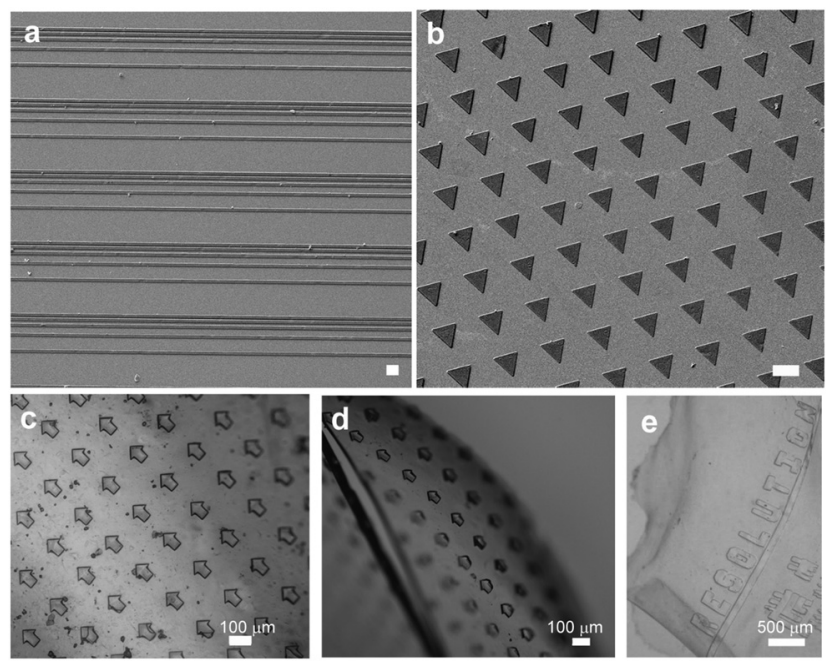

Fig. 3 Images of the microstructures of chitin formed using photolithography (a) and (b) SEM images of high resolution structures (scale bars = $100 \mu \mathrm{m})$ formed on glass substrates. (c) and (d) The patterns $(50 \mu \mathrm{m}$ arrows) can also be formed directly on chitin sheets, leading to flexible micropatterned sheets as seen in (e) with $100 \mu \mathrm{m}$ letters spelling out the word "Resolution". 


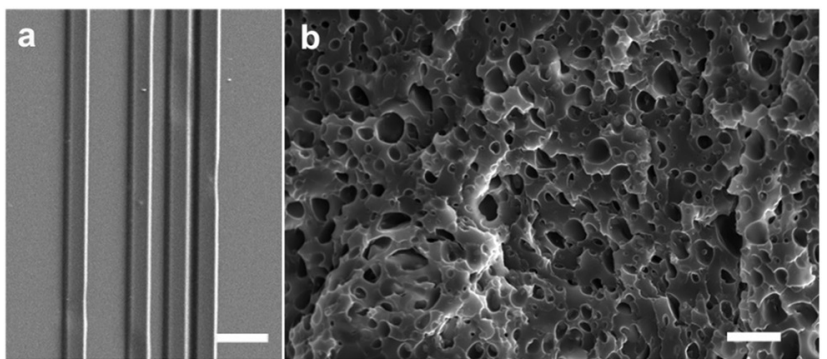

Fig. 4 SEM images of (a) 2D gradient architecture used for cell guidance (scale bar $=50 \mu \mathrm{m}$ ) and $(\mathrm{b})$ the photochitin can be photocrosslinked into a porous 3D hydrogel (scale bar $=5 \mu \mathrm{m}$ ).

features are formed. Owing to the chemical crosslinking between the patterns and the underlying film, these features do not delaminate on mechanical flexure, which is not possible with pure chitin or with micromolding. As above, virtually any kinds of microscale patterns can be formed by simply changing the photomasks. Further examples of the mechanically robust films and patterning are presented in the ESI, $\dagger$ (Fig. S5-S7). By casting the PC solution into a plug followed by photopolymerization, we show the formation of a 3D hydrogels. Using cryofracturing followed by SEM imaging, the interior of the chitin plug is observed to be porous (Fig. 4b). By analyzing several images, the plugs are observed to be microporous with an average pore diameter $\sim 1 \mu \mathrm{m}$. An additional image of the porous surface is shown in Fig. S8 (ESI†). This UV-crosslinkable hydrogel is similar to earlier reports of chitin-based hydrogels. ${ }^{39,40}$ Thus, this photochitin biomaterial can be fashioned into a variety of structures - as micropatterns on rigid substrates, as flexible, micropatterned chitin films, or even as 3D structures, and can be envisioned for various applications including as tissue scaffolds and for wound healing.

\subsection{Cytotoxicity testing and cell guidance}

The ability to provide suitable surfaces that can support cells to carry out necessary functions such as-adhesion, proliferation and differentiation is of great significance in the field of tissue engineering, wound healing, regenerative medicine and cellbased sensing. Bio derived materials are ideal for the realization of such surfaces due to their biocompatible and biodegradable properties. In order to verify that these substrates could be used for tissue engineering applications, films of photochitin were studied for their possible cytotoxicity as well as their suitability for cell culture. Here, we use the terms film and scaffolds interchangeably since these scaffolds are unsupported (free-standing), thin films (patterned or plain) of chitin.

Initially, the fibroblast seeding efficiency was assessed using NIH 3T3 fibroblasts seeded onto scaffolds for $48 \mathrm{~h}$. Patterned scaffolds represent PC films with a gradient pattern $(25 \mu \mathrm{m}$ lines $)$
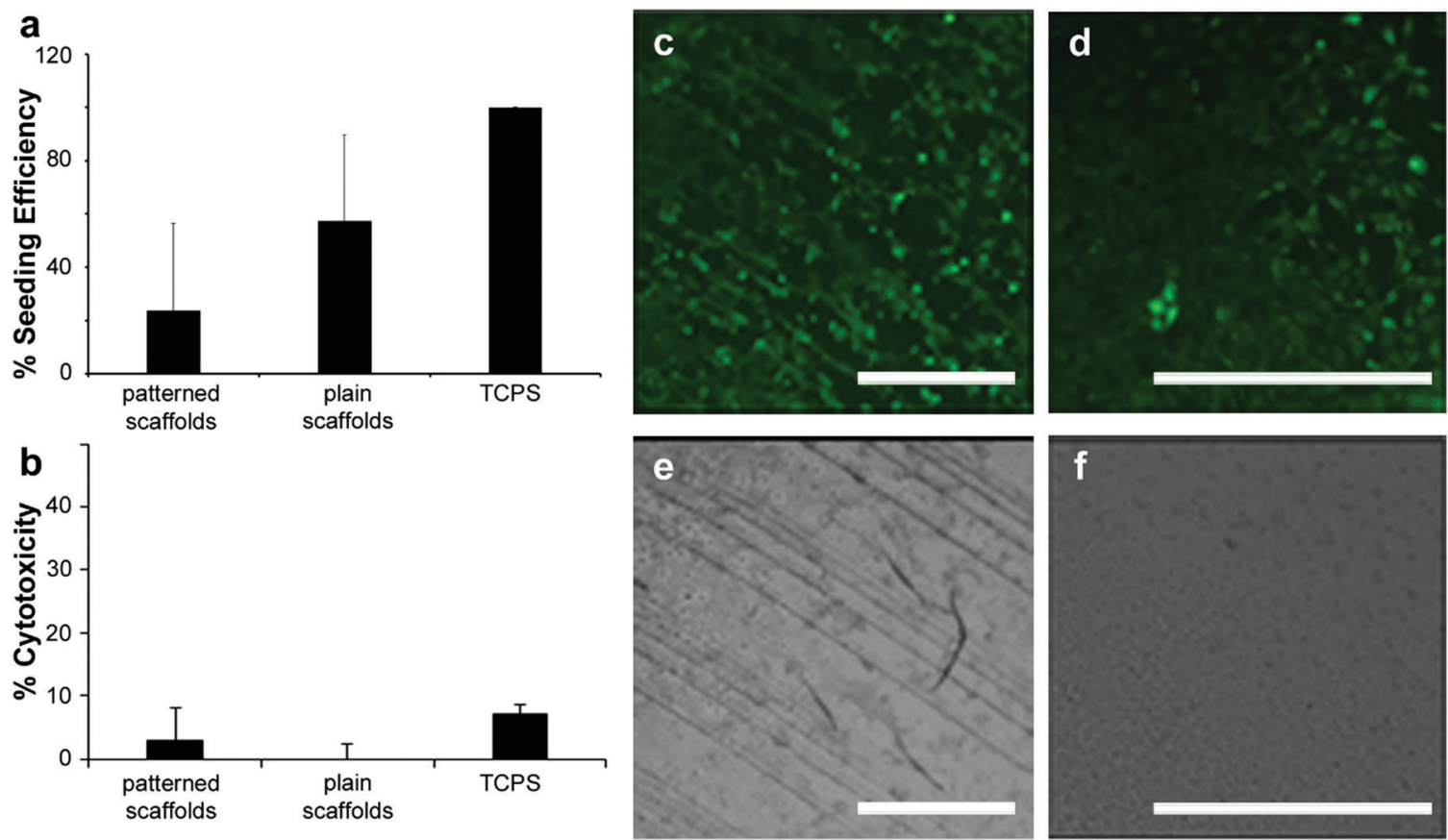

Fig. 5 (a) Fibroblast seeding efficiency. NIH $3 \mathrm{~T} 3$ fibroblasts seeded onto scaffolds for $48 \mathrm{~h}$. MTT viability assay run on scaffolds and compared to $3 \mathrm{T3}$ cell standard curve to determine cell number. Patterned scaffolds represent PC films with a gradient pattern as seen in (e) and Fig. 3c. Plain scaffolds have no patterns. Seeding efficiency was determined by normalizing cell number on scaffolds to 10000 cells seeded. (b) Scaffold cytotoxicity. NIH 3T3 fibroblasts co-cultured with scaffolds with the highest seeding efficiency: patterned PC scaffolds (with $25 \mu \mathrm{m}$ gradient lines) and plain PC films $24 \mathrm{~h}$. LDH assay was performed to determine cytotoxicity, where \% cytotoxicity was determined by normalizing scaffolds values to a no scaffold control incubated with lysis buffer. Scaffolds are not cytotoxic to fibroblasts. Data represented as mean \pm standard deviation. (c) Cell guidance on scaffolds. Fluorescence (c and d) and bright field (e and f) images of NIH 3 T3 fibroblasts seeded onto PC + PEG + DA patterned scaffolds (c and e) and plain PC + PEG + DA films (d and f) for $72 \mathrm{~h}$ and stained with cell tracker dye. Fibroblasts appear to grow preferentially along the PC + PEG + DA patterns compared to the plain film surface ( $d$ and f). Scale bar is $500 \mu \mathrm{m}$ on all panels. Panels $d$ and $f$ are shown enlarged since the unpatterned films are essentially featureless. 
as seen in Fig. 4a, whereas plain scaffolds have no patterns. Both scaffolds were fabricated using the same starting material composition. An MTT viability assay was run on the scaffolds and compared to 3T3 cell standard curve to determine cell number. The seeding efficiency was determined by normalizing cell number on scaffolds to 10000 cells seeded (Fig. 5a) (results for 2500 and 5000 cells are shown in the ESI, $\dagger$ Fig. S9). Cytotoxicity was assessed by co-culturing NIH 3T3 fibroblasts with scaffolds with the highest seeding efficiency over $24 \mathrm{~h}$. An LDH assay was performed to determine cytotoxicity, where \% cytotoxicity was determined by normalizing scaffolds values to a no scaffold control incubated with lysis buffer. No apparent cytotoxicity was seen for chitin films co-cultured with NIH 3T3 fibroblasts with standard tissue culture treated polystyrene used as the control.

Fibroblasts were able to adhere to and grow on both patterned and plain films, but there appears to be a slight increase in seeding efficiency on patterned films (Fig. 5b). Lastly, the chitin films provide contact guidance for the fibroblasts as seen by the arrangement of cells along the patterned lines using fluorescent microscopy (Fig. 5c-f). Fluorescence and bright field images of the fibroblasts seeded on patterned and plain films for $72 \mathrm{~h}$ and stained with cell tracker dye, show that the fibroblasts appear to grow preferentially along the patterns in comparison to the plain film surfaces which show cells spreading randomly. Given that chitin is known to be biodegradable, ${ }^{58}$ these flexible micropatterned cell substrates present versatile surfaces that are cell-friendly and can be used for directed growth of cells for various applications in tissue engineering and medicine. The degradability of chitin via enzymes such as chitinase can also be exploited for use of chitin as degradable components in biosensors and electronic devices. ${ }^{65}$ In such systems, the chitin may form structural components (e.g. biocompatible electronic skins), or functional components (e.g. optical devices). ${ }^{33,66}$ As natureinspired structural and functional components, such biomaterials can therefore be used in flexible and green electronics. ${ }^{28,67}$

\section{Conclusions}

We have demonstrated the synthesis and application of a novel photocrosslinkable derivative of chitin which can be photopatterned into 2D and 3D structures. The photochitin can form optically transparent, mechanically robust films and 3D plugs that are easily formed using a benign solvent. High-resolution microstructures can be patterned on rigid substrates such as glass or silicon, as well as form flexible micropatterned sheets. These micropatterned sheets are non-cytotoxic, and can function as substrates for the controlled guidance of cells. Chitin is biodegradable owing to the action of chitinase enzymes that are widely found in nature. Thus, this biomaterial can form functional components of tissue scaffolds or bioelectronics that can be fully degraded. As an abundantly available renewable resource and byproduct of the food-processing industry, large quantities of this chitin are produced annually. Providing new avenues for its use in biomedical applications and bioelectronics can be envisioned from this material.

\section{Conflicts of interest}

There are no conflicts to declare.

\section{Acknowledgements}

This research was partly supported by funding from the National Science Foundation (CBET-1704435). We thank Dr Meng $\mathrm{Xu}$ for help with SEM images that were obtained at the VCU Nanomaterials Characterization Center. K. M. Moore was supported by a National Science Foundation Graduate Research Fellowship under Grant No. DGE-1650116.

\section{References}

1 M. Rinaudo, Prog. Polym. Sci., 2006, 31, 603-632.

2 R. Jayakumar, D. Menon, K. Manzoor, S. V. Nair and H. Tamura, Carbohydr. Polym., 2010, 82, 227-232.

3 D. Elieh-Ali-Komi and M. R. Hamblin, Int. J. Adv. Res., 2016, 4, 411.

4 M. Božič, S. Gorgieva and V. Kokol, Carbohydr. Polym., 2012, 87, 2388-2398.

5 G.-y. Li, K.-l. Huang, Y.-r. Jiang, P. Ding and D.-l. Yang, Biochem. Eng. J., 2008, 40, 408-414.

6 H. Bao, Y. Pan, Y. Ping, N. G. Sahoo, T. Wu, L. Li, J. Li and L. H. Gan, Small, 2011, 7, 1569-1578.

7 S. V. Madihally and H. W. T. Matthew, Biomaterials, 1999, 20, 1133-1142.

8 M. N. V. Ravi Kumar, React. Funct. Polym., 2000, 46, 1-27.

9 E. Khor and L. Y. Lim, Biomaterials, 2003, 24, 2339-2349.

10 A. Wang, W. Cao, K. Gong, Q. Ao, K. Jun, C. He, Y. Gong and X. Zhang, Asian Chitin J., 2006, 2, 53-60.

11 D. L. Nettles, S. H. Elder and J. A. Gilbert, Tissue Eng., 2002, 8, 1009-1016.

12 R. Jayakumar, M. Rajkumar, H. Freitas, N. Selvamurugan, S. V. Nair, T. Furuike and H. Tamura, Int. J. Biol. Macromol., 2009, 44, 107-111.

13 H. Jiankang, L. Dichen, L. Yaxiong, Y. Bo, Z. Hanxiang, L. Qin, L. Bingheng and L. Yi, Acta Biomater., 2009, 5, 453-461.

14 X. Li and Q. Feng, Polym. Bull., 2005, 54, 47-55.

15 K. Madhumathi, P. T. Sudheesh Kumar, K. C. Kavya, T. Furuike, H. Tamura, S. V. Nair and R. Jayakumar, Int. J. Biol. Macromol., 2009, 45, 289-292.

16 K. Madhumathi, P. T. Sudheesh Kumar, S. Abhilash, V. Sreeja, H. Tamura, K. Manzoor, S. V. Nair and R. Jayakumar, J. Mater. Sci.: Mater. Med., 2010, 21, 807-813.

17 K. E. Park, S. Y. Jung, S. J. Lee, B. M. Min and W. H. Park, Int. J. Biol. Macromol., 2006, 38, 165-173.

18 Z. Huang, Q. L. Feng, B. Yu and S. J. Li, Mater. Sci. Eng., C, 2011, 31, 683-687.

19 N. L. B. M. Yusof, A. Wee, L. Y. Lim and E. Khor, J. Biomed. Mater. Res., 2003, 66A, 224-232.

20 P. R. Marreco, P. da Luz Moreira, S. C. Genari and A. M. Moraes, J. Biomed. Mater. Res., Part B, 2004, 71, 268-277.

21 K. Madhumathi, N. S. Binulal, H. Nagahama, H. Tamura, K. T. Shalumon, N. Selvamurugan, S. V. Nair and R. Jayakumar, Int. J. Biol. Macromol., 2009, 44, 1-5. 
22 H. Tamura, T. Furuike, S. V. Nair and R. Jayakumar, Carbohydr. Polym., 2011, 84, 820-824.

23 A. M. Salaberria, J. Labidi and S. C. Fernandes, Eur. Polym. J., 2015, 68, 503-515.

24 J. Y. Lim and H. J. Donahue, Tissue Eng., 2007, 13, 1879-1891.

25 M. Muskovich and C. J. Bettinger, Adv. Healthcare Mater., 2012, 1, 248-266.

26 R. Chandran, K. Nowlin and D. LaJeunesse, Polymers, 2018, 10, 218.

27 P. Morganti, G. Morganti and M. B. Coltelli, Chitin Nanomaterials and Nanocomposites for Tissue Repair, MarineDerived Biomaterials for Tissue Engineering Applications, Springer, 2019, pp. 523-544.

28 J. Jin, D. Lee, H. G. Im, Y. C. Han, E. G. Jeong, M. Rolandi, K. C. Choi and B. S. Bae, Adv. Mater., 2016, 28, 5169-5175.

29 J. Fukuda, A. Khademhosseini, Y. Yeo, X. Y. Yang, J. Yeh, G. Eng, J. Blumling, C. F. Wang, D. S. Kohane and R. Langer, Biomaterials, 2006, 27, 5259-5267.

30 J. M. Karp, Y. Yeo, W. L. Geng, C. Cannizarro, K. Yan, D. S. Kohane, G. Vunjak-Novakovic, R. S. Langer and M. Radisic, Biomaterials, 2006, 27, 4755-4764.

31 J. G. Fernandez, C. A. Mills and J. Samitier, Small, 2009, 5, 614-620.

32 G. Kumar, Y. C. Wang, C. Co and C. C. Ho, Langmuir, 2003, 19, 10550-10556.

33 P. Hassanzadeh, M. Kharaziha, M. Nikkhah, S. R. Shin, J. Jin, S. He, W. Sun, C. Zhong, M. R. Dokmeci, A. Khademhosseini and M. Rolandi, J. Mater. Chem. B, 2013, 1, 4217-4224.

34 S. Suzuki and Y. Teramoto, Biomacromolecules, 2017, 18, 1993-1999.

35 C. Zhong, A. Kapetanovic, Y. Deng and M. Rolandi, Adv. Mater., 2011, 23, 4776-4781.

36 M. Irimia-Vladu, Chem. Soc. Rev., 2014, 43, 588.

37 J. Huang, Y. Zhong, L. Zhang and J. Cai, Adv. Funct. Mater., 2017, 27, 1701100.

38 K. Ono, Y. Saito, H. Yura, K. Ishikawa, A. Kurita, T. Akaike and M. Ishihara, J. Biomed. Mater. Res., Part B, 2000, 49, 289-295.

39 E. Khor, H. Wu, L. Y. Lim and C. M. Guo, Materials, 2011, 4, 1728-1746.

40 S. Tanodekaew, M. Prasitsilp, S. Swasdison, B. Thavornyutikarn, T. Pothsree and R. Pateepasen, Biomaterials, 2004, 25, 1453-1460.

41 J. A. Rupley, Biochim. Biophys. Acta, 1964, 83, 245-255.

42 M. R. Kasaai, J. Agric. Food Chem., 2009, 57, 1667-1676.

43 T. A. Khan, K. K. Peh and H. S. Ch'ng, J. Pharm. Pharm. Sci., 2002, 5, 205-212.

44 R. Czechowska-Biskup, D. Jarosińska, B. Rokita, P. Ulański and J. M. Rosiak, Prog. Chem. Appl. Chitin Its Deriv., 2012, 17, 5-20.
45 S. Tokura, N. Nishi and J. Noguchi, Polym. J., 1979, 11, 781. 46 K. Azuma, R. Izumi, T. Osaki, S. Ifuku, M. Morimoto, H. Saimoto, S. Minami and Y. Okamoto, J. Funct. Biomater., 2015, 6, 104-142.

47 H. Ehrlich, Extreme biomimetics, Springer, 2017.

48 T. Freier, H. S. Koh, K. Kazazian and M. S. Shoichet, Biomaterials, 2005, 26, 5872-5878.

49 E. S. Gil, S.-H. Park, J. Marchant, F. Omenetto and D. L. Kaplan, Macromol. Biosci., 2010, 10, 664-673.

50 F. Galeotti, A. Andicsova, S. Yunus and C. Botta, Soft Matter, 2012, 8, 4815-4821.

51 A. Revzin, R. J. Russell, V. K. Yadavalli, W.-G. Koh, C. Deister, D. D. Hile, M. B. Mellott and M. V. Pishko, Langmuir, 2001, 17, 5440-5447.

52 N. E. Kurland, T. Dey, S. C. Kundu and V. K. Yadavalli, Adv. Mater., 2013, 25, 6207-6212.

53 O. Agboh and Y. Qin, Polym. Adv. Technol., 1997, 8, 355-365.

54 E. Khor, Chitin: fulfilling a biomaterials promise, Elsevier, 2014.

55 J. Yun, F. Jin, A. Kishita, K. Tohji and H. Enomoto, Formic acid production from carbohydrates biomass by hydrothermal reaction, Journal of Physics: Conference Series, IOP Publishing, 2010, p. 012126.

56 Y. Wu, T. Sasaki, S. Irie and K. Sakurai, Polymer, 2008, 49, 2321-2327.

57 C. Pillai, W. Paul and C. P. Sharma, Prog. Polym. Sci., 2009, 34, 641-678.

58 K. Tomihata and Y. Ikada, Biomaterials, 1997, 18, 567-575.

59 Z. Cui, E. S. Beach and P. T. Anastas, Green Chem. Lett. Rev., 2011, 4, 35-40.

60 J. Jin, M. Song and D. J. Hourston, Biomacromolecules, 2004, 5, 162-168.

61 X. D. Oh, S. Shin, C. Lim and S. D. Hwang, Materials, 2013, 6, 3826-3839.

62 A. Bégin and M.-R. Van Calsteren, Int. J. Biol. Macromol., 1999, 26, 63-67.

63 S. Park, K. Marsh and J. Rhim, J. Food Sci., 2002, 67, 194-197.

64 N. L. B. M. Yusof, L. Y. Lim and E. Khor, Carbohydr. Res., 2004, 339, 2701-2711.

65 W. Suginta, P. Khunkaewla and A. Schulte, Chem. Rev., 2013, 113, 5458-5479.

66 Y.-H. Lin, P.-L. Kang, W. Xin, C.-S. Yen, L.-C. Hwang, C.-J. Chen, J.-T. Liu and S. J. Chang, Comput. Ind., 2018, 100, 1-6.

67 Y. Q. Liu, K. He, G. Chen, W. R. Leow and X. D. Chen, Chem. Rev., 2017, 117, 12893-12941. 